\title{
Fault Characteristic Analysis of Single-Phase Grounding Fault in AC grid of Energy Router in Interacted and Interconnected Micro Grid
}

\author{
Zi-xia Sang ${ }^{1, \text { a }}$, Reng-cun Fang ${ }^{1}$, He Lei ${ }^{2}$, Dong-jun Yang ${ }^{2}$, Zhu Chen², and Jiong Yan ${ }^{2}$ \\ ${ }^{1}$ State Grid HBEPC Economic \& Technology Research Institute, Wuhan 430077, China \\ ${ }^{2}$ State Grid Laboratory for Hydro-thermal Power Resources Optimal Allocation \& Simulation Technology, Wuhan 430077, China
}

\begin{abstract}
The energy router composed of double active bridge (DAB) can interconnect multiple voltage level AC distribution network through DC lines to form AC/DC hybrid distribution network. The fault types of the system are diverse and the fault characteristics are complex. This paper studies the fault characteristics of the system in detail. The single-phase grounding fault of AC network side and singlephase grounding fault of $\mathrm{AC}$ valve side occurred in the system. This paper explored the impact of the AC side of the system after the above failure, and make a detailed comparative analysis. The results show that $\mathrm{DAB}$ can isolate the influence of $\mathrm{AC}$ side fault, and provide theoretical basis for the selection of grounding mode and the design of protection system for $\mathrm{AC} / \mathrm{DC}$ hybrid distribution network.
\end{abstract}

\section{Introduction}

In recent years, with the aggravation of energy shortage and environmental pollution in the world, distributed energy technology has developed rapidly. DC power grid is easier to realize distributed energy access than traditional AC power grid, and has the advantages of low loss, low environmental pollution and high power quality, so it has been widely concerned and studied [1-2]. From the perspective of using the existing $\mathrm{AC}$ distribution network resources and the advantages of DC distribution network, AC / DC hybrid distribution network will be one of the important forms of future distribution network [3]. The United States, Japan, Italy and other countries have successively carried out research on AC / DC hybrid distribution network, and proposed their respective typical system structures [4-5]. In recent years, China has begun to study system control strategy, topology design and other aspects [6-7].

It is one of the common schemes to connect multiple AC distribution networks through DC distribution network by converter stations. However, due to the different $\mathrm{AC}$ distribution network voltage level is not consistent, the DC bus voltage level formed by converter station will be different. In order to realize the interconnection of DC buses with different voltage levels, a feasible method is to unify the voltage levels of DC buses through energy routers, and then interconnect various systems. At present, there are three types of energy routers commonly used: isolated type, partially isolated type and non isolated type [8]. However, energy router applied in AC / DC hybrid distribution network needs to realize electrical isolation. Therefore, this paper selects the dual active bridge (DAB) energy router suitable for medium and high power applications as the intermediate equipment of interconnected $\mathrm{AC}$ distribution network and DC distribution network.

\section{AC network side fault analysis}

Taking the single-phase grounding fault of high-voltage AC network side as an example, the equivalent circuit of high-voltage AC system after fault is shown in Figure 1. After the single-phase grounding fault of phase A, the voltage and current at the network side of the highvoltage AC system will appear negative sequence and zero sequence components. Because the transformer adopts Y-delta connection method, the zero sequence circuit only exists at the network side of the transformer, as shown in the dotted line in Figure 1.

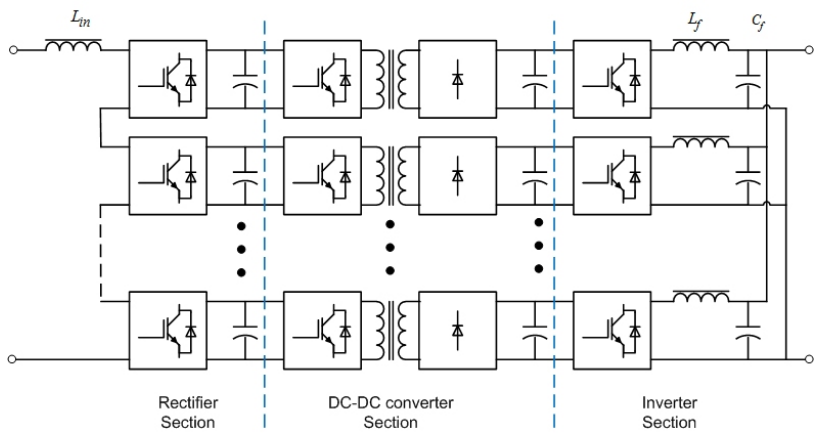

Figure 1. Equivalent circuit of the high-voltage AC grid side.

According to the fault characteristics of AC distribution network, the voltage of phase a decreases and the current increases, while the fault current of phase $\mathrm{B}$ and phase $\mathrm{C}$ is 0 . The negative sequence components

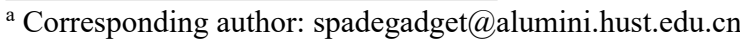


of AC system voltage and current can be transmitted to the VSC inlet through the transformer, which will affect the voltage and current of the high voltage DC side. Let the fundamental frequency component of the current switching function of VSC be:

$$
\left\{\begin{array}{c}
S_{i a}=S_{m} \sin \left(\omega t+\theta_{s}\right) \\
S_{i a}=S_{m} \sin \left(\omega t+\theta_{s}-\frac{2 \pi}{3}\right) \\
S_{i a}=S_{m} \sin \left(\omega t+\theta_{s}+\frac{2 \pi}{3}\right)
\end{array}\right.
$$

Since the negative sequence component of grid side current can be transmitted to the AC side of VSC through the transformer, the AC side current of VSC is composed of positive sequence component and negative sequence component after fault, and the three-phase current at AC side of VSC can be expressed as:

$$
\left\{\begin{array}{c}
i_{s a}=I_{m}^{+} \sin \left(\omega t+\theta^{+}\right)+I_{m}^{-} \sin \left(\omega t+\theta^{-}\right) \\
i_{s b}=I_{m}^{+} \sin \left(\omega t+\theta^{+}-\frac{2 \pi}{3}\right)+I_{m}^{-} \sin \left(\omega t+\theta^{-}+\frac{2 \pi}{3}\right) \\
i_{s c}=I_{m}^{+} \sin \left(\omega t+\theta^{+}+\frac{2 \pi}{3}\right)+I_{m}^{-} \sin \left(\omega t+\theta^{-}-\frac{2 \pi}{3}\right)
\end{array}\right.
$$

Then the DC side current of VSC is:

$$
i_{d c h}=S_{i a} i_{s a}+S_{i b} i_{s b}+S_{i c} i_{s c}
$$

It can be transferred as:

$$
i_{d c h}=\frac{3}{2} S_{m} i_{m}^{+} \cos \left(\theta_{s}-\theta^{+}\right)-\frac{3}{2} S_{m} i_{m}^{-} \cos \left(2 \omega t+\theta_{s}+\theta^{-}\right)
$$

After VSC transformation, the AC current with negative sequence component will generate the second harmonic component at the high voltage DC bus of the system, which will cause the double frequency fluctuation of the voltage of the high voltage DC bus. After the DC voltage is modulated, the third harmonic will be generated in the AC side current of VSC. In turn, when the current in VSC AC side contains negative sequence components, the $\mathrm{AC}$ side will generate odd harmonics of 3,5 and 7 times, and even several harmonics of twice, 4th and 6th order on the DC side. The harmonic of AC side and DC side will affect the safe and stable operation of the system, and the harmonic can be suppressed by the design of corresponding control strategy.

After the single-phase grounding fault occurs at the high-voltage AC network side, the simulation waveforms of the phase voltage, the high-voltage DC bus voltage and the low-voltage DC bus voltage of the system are shown in Figure 2.

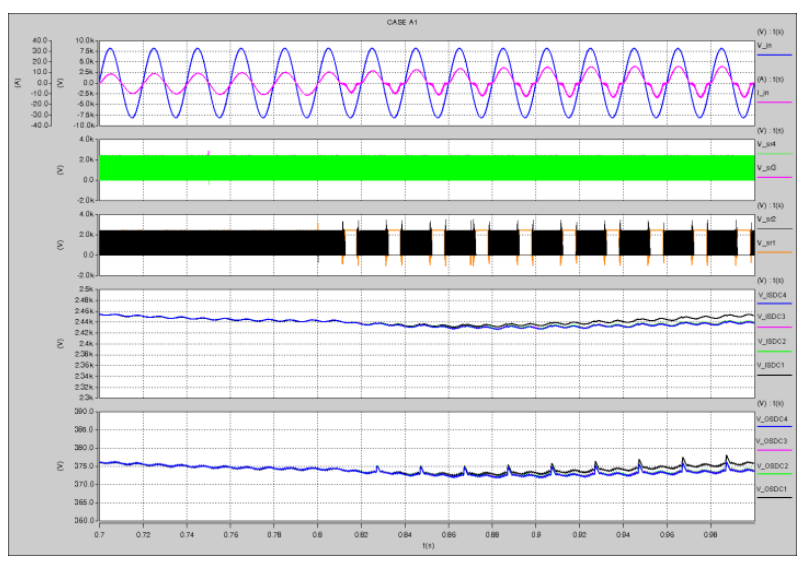

Figure 2. Single-phase grounding fault.

It can be seen that the DC side voltage of VSC can still maintain normal amplitude after AC network side fault occurs. Therefore, the amplitude of DAB output voltage can be kept constant under the action of DAB isolation transformer. The single phase shift control strategy of DAB can reduce the fluctuation of input voltage to a certain extent, reduce the influence of input voltage fluctuation on output voltage, and maintain the stability of low voltage DC bus voltage. Therefore, DAB can isolate the high-voltage AC network side fault, make the low-voltage DC bus and low-voltage AC system unaffected, effectively improve the fault tolerance of AC / DC hybrid distribution network, and reduce the impact of AC system network side fault on DC side.

The single-phase grounding fault at the high-voltage AC network side of the system will cause the voltage and current changes of the high-voltage $\mathrm{AC}$ side and the high-voltage DC side. The A-phase voltage of the highvoltage $\mathrm{AC}$ side drops to 0 , and the three-phase voltage is no longer balanced, resulting in negative sequence and zero sequence components. The voltage of high voltage DC bus fluctuates twice, and the voltage of low voltage DC bus is not affected by grid side fault.

To sum up, the impact of high-voltage AC network side fault on the high-voltage DC system is mainly caused by the double frequency fluctuation of DC voltage. DAB can isolate the high-voltage AC network side fault, ensure that the low-voltage DC side and lowvoltage AC side of the system will not be affected after the fault, reduces the requirements for the insulation level of the low-voltage system, and maintains the stable operation of the low-voltage system.

\section{AC VSC side fault analysis}

Taking the single-phase grounding fault of high-voltage $\mathrm{AC}$ valve side as an example, after the fault occurs, the voltage and current at the AC side of VSC will appear negative sequence and zero sequence components, but due to the isolation effect of transformer, the zero sequence component will not be transmitted to the network side AC system. The influence mechanism of negative sequence component on the system is the same as that of high voltage AC network side fault. The influence of zero sequence component on system voltage 
and current characteristics is closely related to the DC side capacitive grounding mode of VSC. When the neutral point of VSC DC side capacitor is not grounded, there is no zero sequence current path.

After the fault occurs, the zero sequence current path is composed of the fault point, VSC and DC capacitor grounding point, and the positive currents of HVDC bus can be given by:

$$
i_{d c h p}=S_{i a} i_{a}+S_{i b} i_{b}+S_{i c} i_{c}
$$

It can be transferred as:

$$
\begin{aligned}
& i_{d c h p}=\frac{1}{2}\left(S_{i a}^{+} i_{a}^{+}+S_{i b}^{+} i_{b}^{+}+S_{i c}^{+} i_{c}^{+}+S_{i a}^{-} i_{a}^{-}+S_{i b}^{-} i_{b}^{-}+S_{i c}^{-} i_{c}^{-}\right. \\
& +\frac{1}{2}\left(S_{i a}^{-} i_{a}^{+}+S_{i b}^{-} i_{b}^{+}+S_{i c}^{-} i_{c}^{+}+S_{i a}^{+} i_{a}^{-}+S_{i b}^{+} i_{b}^{-}+S_{i c}^{+} i_{c}^{-}\right)+\frac{3}{2} i^{0}
\end{aligned}
$$

The negative currents of HVDC bus can be given by:

$$
i_{d c h m}=\left(S_{i a}-1\right) i_{a}+\left(S_{i b}-1\right) i_{b}+\left(S_{i c}-1\right) i_{c}
$$

It can be transferred as:

$$
\begin{aligned}
& i_{d c h m}=\frac{1}{2}\left(S_{i a}^{+} i_{a}^{+}+S_{i b}^{+} i_{b}^{+}+S_{i c}^{+} i_{c}^{+}+S_{i a}^{-} i_{a}^{-}+S_{i b}^{-} i_{b}^{-}+S_{i c}^{-} i_{c}^{-}\right. \\
& +\frac{1}{2}\left(S_{i a}^{-} i_{a}^{+}+S_{i b}^{-} i_{b}^{+}+S_{i c}^{-} i_{c}^{+}+S_{i a}^{+} i_{a}^{-}+S_{i b}^{+} i_{b}^{-}+S_{i c}^{+} i_{c}^{-}\right)-\frac{3}{2} i^{0}
\end{aligned}
$$

The zero sequence current produces a common mode component in the positive and negative currents of the DC side of the VSC, but the common mode component does not exist in the inter electrode voltage.

Therefore, there is a fundamental common mode component in the positive and negative current of $\mathrm{DAB}$ input side, but this common mode component does not exist in the voltage between electrodes. Similarly, after the adjustment of DAB single phase-shifting control strategy, the second harmonic fluctuation can be suppressed, and the impact of HVDC fault on lowvoltage DC system and low-voltage AC system will be reduced.

After single-phase grounding fault at high-voltage AC VSC side, the voltage waveform of high-voltage DC bus and low-voltage DC bus is shown in Figure 3.

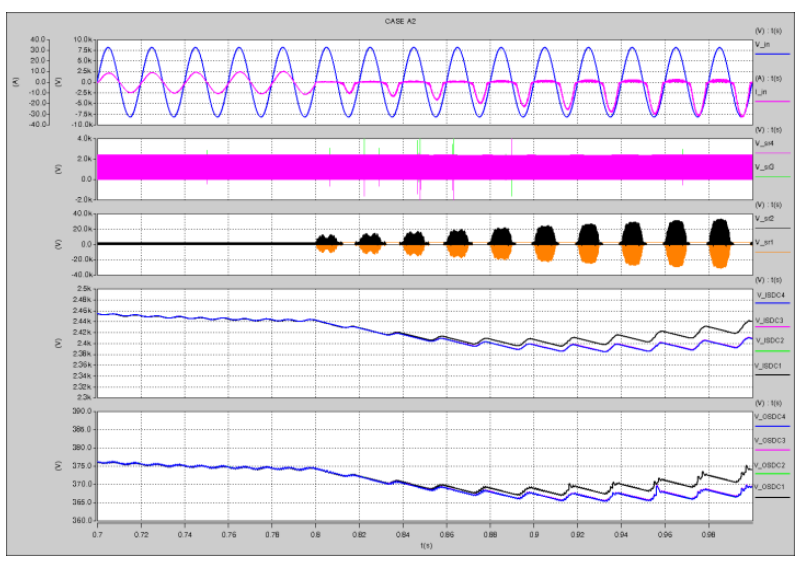

Figure 3. Single-phase grounding fault at high-voltage AC VSC side.
It can be seen that after the single-phase grounding fault at the high-voltage AC valve side, the voltage at the positive and negative sides of the high-voltage DC side will fluctuate in the common mode at the fundamental frequency, but the common mode fluctuation will not affect the characteristics of the DC inter electrode voltage. The common mode fluctuation can be suppressed by DAB based energy router, as shown in Figure 4.

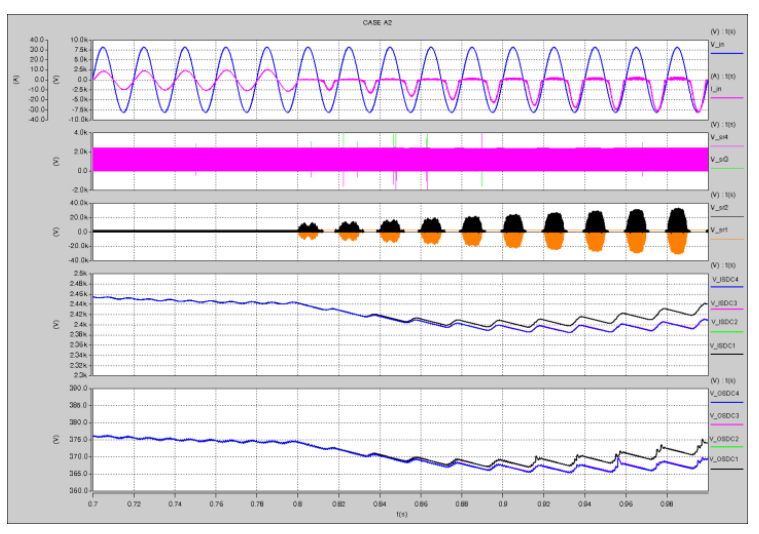

Figure 4. Single-phase grounding fault for bus voltage.

Constant active power control strategy is adopted for VSC at low voltage side of the system. After singlephase grounding fault occurs at AC VSC side, the characteristics of voltage and current at AC side of VSC are similar to those of high-voltage AC VSC side. It can be seen that $\mathrm{DAB}$ energy router can isolate $\mathrm{AC}$ system from DC system, ensure that AC system fault will not be transmitted to DC side, and maintain normal operation of DC system after AC fault.

\section{Conclusions}

In this paper, the existence of $\mathrm{DAB}$ can isolate the influence of high-voltage and low-voltage AC system faults on the non fault side system, and maintain the stability of DC bus voltage on the non fault side. When high voltage AC network side fault occurs in the system, $\mathrm{DAB}$ can isolate the double frequency fluctuation of high voltage DC bus to ensure that the low voltage DC bus will not be affected in the process of fault. When high voltage $\mathrm{AC}$ valve side fault occurs in the system, the DC bus on the corresponding fault side will have common mode fluctuation. DAB can also suppress the common mode fluctuation of DC bus on the non fault side, and avoid the fault transmission from $\mathrm{AC}$ valve side of high voltage and low voltage side to non fault system. Therefore, DAB energy router not only realizes the interconnection of multiple voltage levels AC distribution network, but also avoids the mutual influence between fault system and non fault system after AC side fault, so as to ensure the normal operation of non fault system. This characteristic greatly improves the reliability of the system and reduces the complexity of control strategy and protection system design of $\mathrm{AC} /$ DC system. 


\section{Acknowledgments}

This research was supported in part by the State Grid Hubei Electric Power Company Science and Technology Project (52153820000H).

\section{References}

1. W. Sheng, R. Li, Y. Li, et al. Proceedings of the CSEE, 36, 3391-3403 (2016).

2. Q. Song, B. Liu, W. Liu, et al. Proceedings of the CSEE, 33, 9-19 (2013).

3. R. Huang, T. Pu, K. Liu, et al. Automation of Electric Power Systems, 39, 26-33 (2015).

4. H. Kakigano, Y. Miura, T. Ise. IEEE Transactions on Power Electronics, 25, 3066-3075 (2010)

5. D. Jiang, H. Zheng. Automation of Electric Power Systems, 36, 98-104 (2012).

6. L. Zhang, W. Tang, J. Liang, et al. Proceedings of the CSEE, 36, 6067-6075 (2016).

7. H. Wu, J. Zhang, Y. Xing. IEEE Transactions on Power Electronics, 30, 735-746 (2015). 